\title{
Evaluation des estimations indirectes de mortalité dans trois observatoires de population au Sénégal
}

\section{Bruno Masquelier ${ }^{1}$ a, b, Cheikh Tidiane Ndiaye ${ }^{c}$, Gilles Pison ${ }^{b, d}$, Ndeye Binta Dieme ${ }^{c}$, Ibrahima DIOUF c, Stéphane Helleringer ${ }^{e}$, Ousmane Ndiaye ${ }^{f}$ et Valérie Delaunay ${ }^{g}$}

a Centre de recherche en démographie, Université catholique de Louvain, Louvain-la-Neuve, Belgique

b Institut National d'Etudes Démographiques, Paris, France

c Agence Nationale de la Statistique et de la Démographie, Dakar, Sénégal

d Laboratoire d'éco-anthropologie et ethnobiologie, Muséum national d'histoire naturelle, Paris, France

e Johns Hopkins University, Bloomberg School of Public Health, Baltimore, USA

f URMITE, UMR198 AMU-IRD, Institut de recherche pour le développement (IRD), Dakar Sénégal

g LPED UMR151 AMU-IRD, Institut de recherche pour le développement (IRD), Dakar, Sénégal

\begin{abstract}
In Sub-Saharan Africa, censuses are a key source of data for estimating mortality because death registration is incomplete. To assess the reliability of census-based mortality estimates, we apply different methods to individual-level data extracted from the 2002 and 2013 Senegalese censuses covering three health and demographic surveillance systems (HDSS) in Bandafassi Mlomp and Niakhar. The under-five mortality rates inferred from reports on children ever born and surviving are lower than expected based on longitudinal demographic surveillance. Estimates derived from reports on parental survival are also much lower than the adult mortality levels observed in HDSS field sites. By contrast, age-specific death rates based on recent deaths reported in households are consistent with HDSS data, except for infant mortality, which is significantly under-reported in 2002. This evaluation confirms that indirect estimates of mortality obtained from census data should not be considered in isolation but must instead be systematically compared with each other. Direct evaluation studies conducted at the individual level using record linkages are needed to better identify the various sources of bias.
\end{abstract}

Keywords: Data quality, Sub-Saharan Africa, Senegal, mortality estimation, indirect methods.

\section{Résumé}

En Afrique sub-saharienne, les recensements constituent une source de données fondamentale pour estimer la mortalité, en raison de l'incomplétude de l'enregistrement des décès à l'état civil. Afin d'évaluer la fiabilité des niveaux de mortalité qui peuvent en être déduits, nous appliquons différentes méthodes d'estimation à des extraits des recensements sénégalais de 2002 et 2013, couvrant trois observatoires de population situés à Bandafassi, Mlomp et Niakhar. Les taux de mortalité des moins de cinq ans tirés du nombre d'enfants nés vivants et survivants s'avèrent plus bas que les niveaux attendus au vu des données du suivi démographique. Les estimations déduites des déclarations sur la survie des parents sont largement inférieures aux niveaux de mortalité adulte réellement observés selon le suivi. Par contre, les taux de mortalité par âge basés sur les décès récents déclarés dans les ménages sont

\footnotetext{
${ }^{1}$ Bruno Masquelier

Centre de recherche en démographie/Centre for Demographic Research, Université catholique de Louvain,

Place Montesquieu 1, Boîte L2.08.03, Louvain-la-Neuve, 1348, Belgique

Tél : +3210472617

E-mail : bruno.masquelier@uclouvain.be
} 
conformes aux données du suivi, sauf pour la mortalité infantile qui est sensiblement sous-déclarée en 2002. Cette évaluation confirme que les méthodes indirectes procurent des estimations qui ne peuvent être considérées isolément mais doivent plutôt être systématiquement comparées les unes aux autres. Des études d'évaluation directes menées au niveau individuel à l'aide d'appariements sont nécessaires pour mieux identifier les différentes sources de biais.

Mots-clés: Qualité des données, Afrique sub-saharienne, Sénégal, estimation de la mortalité, mesures indirectes

\section{Introduction}

Des données récentes et fiables sur la mortalité sont essentielles pour identifier les priorités en matière de santé publique et orienter l'allocation des ressources dans ce secteur. Or, en l'absence d'un enregistrement complet des décès à l'état civil, la plupart des pays d'Afrique sub-saharienne doivent s'appuyer sur leurs recensements et des enquêtes par sondage pour estimer les risques de décès. Le Sénégal ne fait pas exception; selon le dernier recensement de 20/3, seuls $35 \%$ des décès sont déclarés à l'état civil. Dans ce contexte, il est important d'évaluer la qualité des données recueillies de façon rétrospective dans les enquêtes et les recensements. Les recensements sont tout particulièrement sujets à des erreurs de couverture et de contenu, en raison de l'ampleur et de la complexité de ces opérations. Les erreurs de couverture peuvent comprendre des sousdénombrements sélectifs de certains résidents (tels que les enfants ou les jeunes migrants), des doubles comptages ou des omissions d'individus. Les erreurs de contenu peuvent également prendre différentes formes, telles que les déclarations erronées de l'âge des individus, des omissions de certains décès, ainsi que des erreurs de classification de décès liés ou non à une grossesse et des erreurs sur leur datation. Ces différents types d'erreurs sont tous susceptibles de biaiser les niveaux de mortalité obtenus, mais leurs effets combinés sont difficiles à prévoir. De nombreuses méthodes démographiques ont été développées pour détecter et tenir compte de ces erreurs, notamment par le biais d'un examen de la validité externe ou de la cohérence interne des estimations (Ewbank 198I, Moultrie et al. 2013). La comparaison entre différentes sources de données est l'une des voies les plus indiquées, mais il est bien souvent difficile d'aboutir à des conclusions définitives en l'absence d'une référence qui soit elle-même peu entachée d'erreurs.

Dans cet article, nous utilisons comme base de comparaison les niveaux de mortalité déduits des données longitudinales recueillies dans trois observatoires de population au Sénégal, dans les zones rurales de Bandafassi, Mlomp et Niakhar. Nous dégageons une série d'estimations indirectes à partir des deux recensements nationaux de 2002 et 2013, dont nous avons extrait les données relatives uniquement aux villages qui font l'objet d'une surveillance démographique. Nous examinons (I) les tendances de la mortalité des moins de cinq ans obtenues des déclarations sur les enfants nés vivants et décédés, (2) les quotients de mortalité entre 15 et 60 ans estimés à partir des déclarations sur la survie des parents, ainsi que (3) les tables de mortalité déduites des informations sur les décès récents des membres des ménages et les survivants par âge et sexe.

\section{Revue de littérature}

Selon le dernier recensement, la population sénégalaise bénéficiait d'une espérance de vie de 65 ans en 2013, l'une des plus élevées en Afrique subsaharienne. Entre 2000 et 2015 , cette espérance de vie a progressé de 8.7 ans, soit une hausse de près de 7 mois chaque année (United Nations 2015). Ces progrès sont surtout portés par la baisse rapide de la mortalité des enfants. Même si les niveaux actuels restent élevés ( 55 décès avant 5 ans pour 1000 naissances en $2013^{2}$ ), le Sénégal a manqué de peu la cible fixée par les Objectifs du Millénaire pour le Développement (OMD 4). Par contre, la mortalité a évolué moins vite aux âges adultes, bien que le pays ait été relativement épargné par le $\mathrm{VIH} / \mathrm{sida}$ (Masquelier etal. 2014). Un jeune de 15 ans a toujours une probabilité d'environ un cinquième de décéder avant d'atteindre 60 ans. L'objectif de réduction de trois quarts du taux de mortalité maternelle (OMD 5) est donc loin d'être atteint (ce taux s'élevait encore à 434 décès pour 100000 naissances vivantes en $\left.2013^{3}\right)$. En raison de ces progrès variables, la répartition par âge des décès est en train de changer: en 1980-1985, environ la moitié des décès concernaient des enfants de moins 5 ans, contre moins d'un tiers aujourd'hui. Pour suivre ces évolutions démographiques, le Sénégal a besoin de disposer d'estimations précises sur les niveaux, les

\footnotetext{
2 Sc: Estimations du Groupe Inter-Agences des Nations Unies pour l'estimation de la mortalité des enfants: http://childmortality.org/

${ }^{3}$ Sc : Rapport définitif du RGPHAE 2013. En 2011, l'enquête EDS situait cet indicateur à 409 selon l'estimation directe et 309 selon l'estimation indirecte.
} 
tendances et les différentiels de la mortalité, et ce y compris aux âges adultes.

Pour les enfants, le suivi de la mortalité a été grandement facilité par la multiplication des enquêtes rétrospectives à large échantillon, telles que les Enquêtes Démographiques et de Santé (EDS) et les enquêtes à indicateurs multiples (MICS). Ces enquêtes permettent de recueillir des informations sur l'histoire génésique des femmes en âge de reproduction et sur la survie de leurs enfants. En la matière, le Sénégal se distingue de ces voisins car il dispose aujourd'hui de nombreuses enquêtes permettant de reconstituer les tendances avec précision (Alkema et al. 20/4), notamment une enquête EDS continue. En plus de ces histoires génésiques, les données sur le nombre d'enfants nés vivants et décédés sont également exploitées à l'aide de la méthode indirecte initialement développée par W. Brass (Hill 20I3). Au Sénégal, l'enquête démographique de $1960-6 \mid$, et certaines enquêtes MICS donnent lieu à des estimations indirectes utilisées par les différentes agences des Nations Unies impliquées dans le suivi de la mortalité des enfants (UN Inter-agency Group for Child Mortality Estimation). Par contre, les estimations indirectes issues des recensements de 2002 et 2013 sont exclues, car elles sont considérées comme trop peu fiables ${ }^{4}$.

L'estimation de la mortalité des adultes est nettement moins évidente (Masquelier 2010). Trois catégories de méthodes ont été développées, mais aucune d'elles n'est pleinement satisfaisante. Premièrement, les rapports de survie calculés par cohorte sur une période intercensitaire peuvent être convertis en indices de mortalité (Preston and Bennett 1983). Les estimations obtenues s'avèrent toutefois extrêmement sensibles aux variations dans la complétude de l'enregistrement, aux erreurs d'âges systématiques, ainsi qu'aux migrations. Cette méthode est donc quasiment inutilisable en Afrique sub-saharienne. Deuxièmement, des méthodes de " distribution des décès » ont été mises au point pour rehausser les niveaux de mortalité issus de l'état civil ou des informations procurées par les chefs de ménage sur les décès récents (Dorrington 20/3). Deux méthodes sont généralement utilisées: la generalized growth balance method (Hill 1987) et la synthetic extinct generation method (Bennett and Horiuchi 1984). ${ }^{5}$ Elles reposent sur des hypothèses

\footnotetext{
${ }^{4}$ Sc : http://childmortality.org/.

${ }^{5}$ Ces approches procurent une estimation de la complétude de l'enregistrement des décès relative aux deux recensements. La generalized growth balance method procure également une estimation de la complétude de l'enregistrement de la population lors du second recensement (par rapport au premier).
}

assez lourdes : une population fermée aux migrations, une absence d'erreurs d'âges systématiques et surtout une complétude de l'enregistrement de la population et des décès invariante par âge. Enfin, la troisième catégorie de méthodes se base sur l'exploitation de données relatives à la survie des proches ; c'est-à-dire la survie des parents (Timæus 1992), des frères et sœurs (Masquelier 20I4), et des premiers conjoints (Hill 1977). Dans le cas des recensements, bien souvent seules les informations sur la survie des parents sont recueillies.

Chez les enfants comme les adultes, l'estimation de la mortalité est souvent entachée de biais de sélection. Par exemple, les enfants décédés en raison de conditions sanitaires critiques sont moins susceptibles d'avoir encore une mère survivante pour déclarer leur décès. Les erreurs de déclaration peuvent amplifier l'effet de ces biais sur les estimations : présence de données manquantes, imprécisions sur les âges des personnes recensées ou des personnes décédées, omissions de certains évènements... Les schémas d'erreurs vont avoir un impact variable sur les différentes méthodes (Moultrie et al. 2013). Par exemple, en matière de survie des enfants, c'est l'omission d'enfants décédés qui pose le problème le plus sérieux. En matière de survie des parents, le "biais d'adoption" est le problème principal: certains enquêteurs peuvent avoir tendance à considérer les adultes qui cohabitent avec des enfants comme les parents biologiques de ces derniers, et à s'abstenir de poser les questions sur la parenté réelle (Blacker 1984). Les personnes enquêtées peuvent également apporter des réponses erronées si elles ne comprennent pas que la question se réfère à la survie des parents biologiques (et non aux parents adoptifs). En matière de déclaration des décès des douze derniers mois, les omissions de décès sont aussi identifiées comme la principale source d'erreurs, notamment parce que les décès sur une période de 12 mois restent des événements relativement rares, ce qui n'invite pas à poser systématiquement ces questions dans tous les ménages. Timaeus (199I) considère que ces données sont globalement inutiles, car souvent jusqu'à la moitié des décès peuvent être omis. Les rares estimations publiées de la complétude de la déclaration des décès sont toutefois très variables. Par exemple, Hill et Stanton (20II) estiment qu'environ la moitié des décès féminins ont été déclarés dans les recensements de 1986 et 1996 au Lesotho, tandis qu'environ $85 \%$ des décès féminins ont été déclarés dans les recensements du Zimbabwe 1992 et 2002. Au Sénégal, une tentative d'utilisation des méthodes de distribution des décès sur les recensements de 1988 et de 1976 a procuré des 
résultats jugés tout à fait inattendus et peu plausibles (Pison et al. 1995).

$\mathrm{Ce}$ bref aperçu des différentes méthodes disponibles pour estimer la mortalité en l'absence d'état civil fait ressortir une grande diversité des sources d'erreurs, dont les effets sur les estimations sont souvent difficiles à évaluer. Les sites de surveillance démographique peuvent procurer des données fiables pouvant servir de point de comparaison.

\section{Données et méthodes \\ Les observatoires de population de Bandafassi, Mlomp et Niakhar}

Nous utilisons ici les données longitudinales recueillies dans trois observatoires de population (Pison 2005, Ye et al. 20/2). La population de chacun de ces sites a fait l'objet d'un recensement initial puis a été suivie par enquête à passages répétés. De nouveaux individus peuvent entrer dans la population par la naissance ou l'immigration, et les membres des observatoires peuvent les quitter par émigration ou décès. À chaque passage, les enquêteurs examinent la composition de chaque ménage, vérifient la listes de ses membres présents à la visite précédente et recueillent des informations sur les naissances, décès, mariages, et migrations survenus depuis le passage précédent. Les causes de décès sont déterminées par le biais d'autopsies verbales. Ces autopises sont des entretiens avec questionnaires au cours desquelles des enquêteurs interrogent les proches du défunt pour obtenir des informations sur les conditions du décès et les symptômes de la maladie l'ayant précédé (Adjuik et al. 2006, Desgrées du Loû et al. 1996).

Il y a trois observatoires de population au Sénégal. Ils sont situés à Bandafassi, Mlomp et Niakhar (Graphique I). Ces trois sites ne sont nullement représentatifs des régions dans lesquelles ils se trouvent, mais ils présentent toutefois une variété de profils socio-démographiques contrastés qui sont typiques des zones rurales sénégalaises. La collecte des données est globalement similaire dans ces trois sites $^{6}$.

Le plus large observatoire est situé à proximité de la ville de Niakhar, à $135 \mathrm{~km}$ au sud-est de Dakar (Delaunay et al. 2013). Cet observatoire a été créé en 1962 et couvre une population estimée à 45500

\footnotetext{
${ }^{6}$ A la différence de Niakhar et Mlomp, l'information est recueillie auprès d'informateurs locaux plutôt qu'auprès de chaque chef de ménage à Bandafassi.
}

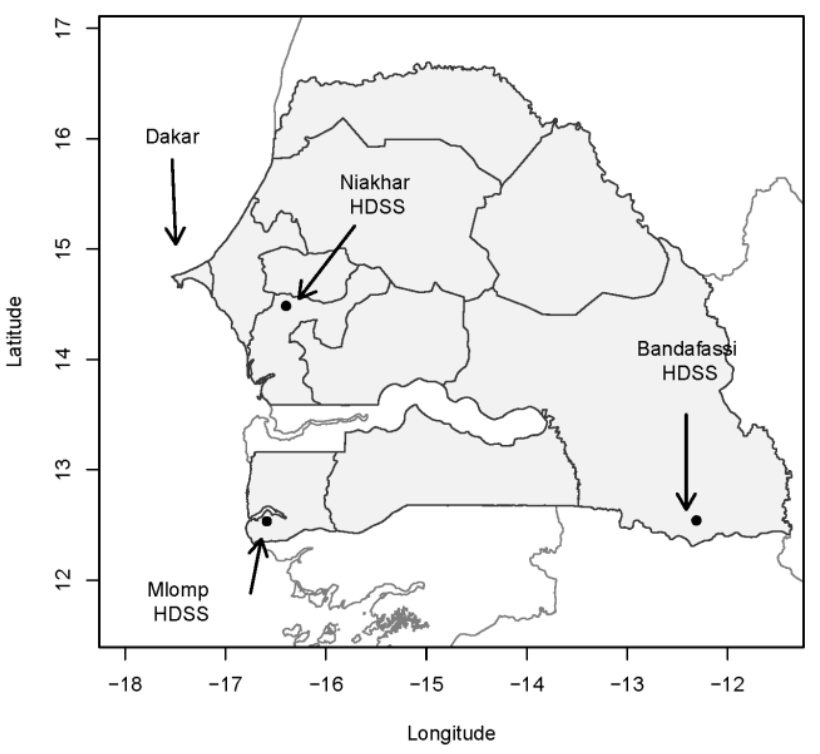

Graphique I : Carte localisant les trois observatoires de population du Sénégal

habitants au moment du recensement de $2013^{7}$. Le groupe ethnique Sereer représente $97 \%$ de la population et il y a également des minorités Wolof, Toucouleur, et Laobe. Les principaux groupes religieux de la région sont les musulmans $(80 \%)$ et les chrétiens (20\%). Le millet est la principale culture vivrière et l'arachide la principale culture de rente. Certains paysans élèvent aussi quelques têtes de bétail. Le climat à Niakhar est typique de la région sahélienne. Les trois plus grands villages de la zone d'étude ont accès à l'électricité et comprennent les établissements de santé, des marchés hebdomadaires, des autobus quotidiens vers Dakar, et plusieurs magasins. Le niveau d'instruction est faible avec $50 \%$ des hommes et $75 \%$ de femmes n'ayant jamais fréquenté l'école primaire. La région est également caractérisée par une fréquence élevée des migrations temporaires et permanentes. Une grande proportion de résidents de Niakhar se déplace à Dakar pour y chercher du travail.

L'observatoire de Bandafassi est situé dans la région de Kédougou, non loin des frontières du Mali et de la Guinée (Pison et al. 20l4). La zone se trouve à $700 \mathrm{~km}$ de Dakar et à $250 \mathrm{~km}$ de Tambacounda, la capitale régionale. La population suivie comprenait environ I 3500 habitants au moment du recensement de 2013, regroupés dans 42 petits villages. La population est divisée en trois groupes ethniques qui vivent dans des villages distincts: les Bedik (qui

\footnotetext{
7 L'observatoire de Niakhar portait au départ sur 65 villages. La zone de suivi démographique a ensuite été réduite à 8 villages en 1969, puis en 1983 elle a été étendue à nouveau pour porter sur les 30 villages suivis actuellement.
} 
représentent $25 \%$ de la population), les Malinkés (16\%) et les Peuls Bande (59\%). L'observatoire a été créé en 1970 à l'origine pour des études génétiques. II ne couvrait au départ que 8 villages habités par un groupe ethnique (les Malinkés) mais il a été étendu ensuite aux autres groupes ethniques en 1975 et 1980. La zone a connu un déclin de mortalité rapide, mais peu de changements sur le plan de la fécondité au cours des trois dernières décennies. La polygamie est fréquente (avec environ 170 femmes mariées pour 100 hommes mariés). La principale activité économique est l'agriculture, avec la culture de céréales (comme le sorgho, le maïs et le riz), les arachides et le coton, ainsi que l'élevage du bétail. Une partie de la population de jeunes hommes effectue des migrations saisonnières vers les villes et d'autres régions rurales, mais la migration est moins fréquente que dans les deux autres sites.

Le dernier observatoire a été créé à Mlomp au sud-ouest du Sénégal en 1985 (Pison et al. 2002). Cet observatoire couvrait une population totale d'environ 8600 résidents au moment du recensement de 2013 , dont la plupart appartiennent à l'ethnie Diola. La population est principalement composée de chrétiens ou d'animistes. En comparaison avec les autres sites, l'infrastructure éducative y est plus développée: plus de la moitié des femmes de 15-49 ans ont déjà été à l'école pendant au moins un an (Duthé 2008). Pendant la saison des pluies, la plupart de la population cultive le riz. Pendant la saison sèche, plus de $40 \%$ de la population adulte migre dans les villes pour travailler comme employé temporaire ou domestique. Les résidents qui restent dans la zone d'étude gagnent de l'argent par la pêche et la production de vin de palme. Ce sont ces résidents qui ont donc rencontré les agents recenseurs, car les deux derniers recensements nationaux ont été organisés durant les mois de novembre et décembre.

\section{Les données des recensements de 2002 et 2013}

Pour évaluer les niveaux de mortalité qui peuvent être déduits des recensements, nous utilisons les données individuelles des deux recensements du Sénégal organisés du 8 au 22 décembre 2002 puis du 19 novembre au 09 décembre 2013. Le questionnaire de ces recensements contenait la plupart des questions couramment utilisées avec des techniques démographiques indirectes : le nombre total d'enfants nés et survivants (par sexe), les naissances des 12 derniers mois, les décès survenus au cours de l'année (par âge et sexe, avec une question supplémentaire pour identifier les décès liés à la grossesse), ainsi que la survie des parents (en 2013). Une différence importante entre le recensement de 2002 et celui de 2013 est que les formulaires papier ont été utilisés en 2002 , tandis que les ordinateurs de poche (PDA) ont été utilisés en 2013. L'introduction de cette innovation technologique visait surtout à raccourcir les délais de publications des résultats et à améliorer la qualité des données grâce aux contrôles de cohérence intégrés et au suivi en temps réel de l'évolution de la collecte.

A aucun moment les agents recenseurs n'ont été mis au courant du fait que cette comparaison allait être menée. Les agents du recensement qui ont travaillé au sein des trois observatoires ont suivi la même formation que l'ensemble des agents impliqués dans le reste du pays.

\section{Méthodes d'analyse}

Les populations résidentes ont d'abord été extraites des bases de données des observatoires à la date des deux recensements pour comparer les effectifs de population, ainsi que les structures par âge et sexe. Les tendances de la mortalité des trois observatoires ont ensuite été estimées à l'aide de méthodes biographiques.

Pour procéder à une comparaison avec les données des recensements, il a d'abord été nécessaire d'identifier avec précision les districts de recensement couvrant les trois zones rurales. La délimitation des localités (villages/hameaux) a donc été harmonisée entre les deux bases de données.

Sur la base des extraits du recensement, les tendances de la mortalité des enfants de moins de cinq ont été obtenues à partir des informations recueillies sur le nombre d'enfants nés vivants et survivants des femmes adultes en 2013 , en utilisant la méthode classique de Trussell (Hill 20/3). Nous avons supposé que la structure par âge de la mortalité était conforme au modèle Sud de tables de mortalité de Coale-Demeny dans les trois sites, après examen de la relation entre la mortalité adulte et celle des enfants. Conformément à une pratique courante, les niveaux déduits des déclarations des plus jeunes mères ( $15-19$ ans) ne sont pas considérés ici. En effet, la méthode d'estimation indirecte repose sur une hypothèse d'invariance de la mortalité selon l'âge des mères, ce qui est rarement vérifié à ces âges.

Les niveaux de mortalité adulte ont été obtenus en appliquant la méthode des orphelins (Brass and Bamgboye 1981, Timæus 1992). Cette méthode consiste à estimer les risques de décès des adultes en interrogeant les descendants. A minima, seules deux informations sont nécessaires : (I) l'âge des individus interrogés et (2) la survie de chacun de leurs parents. Comme la durée d'exposition n'est pas connue, elle est approximée par l'âge des répondants. Une estimation de l'âge moyen à la naissance des enfants est également nécessaire pour tenir compte de l'âge auquel débute en moyenne cette exposition au 
risque. Elle a été déduite des naissances ventilées par âge et survenues dans l'année précédant le recensement. L'âge moyen des pères à la naissance de leurs enfants peut être estimé en ajoutant à celui des mères l'écart d'âge médian entre hommes et femmes mariés. Ici nous exploitons uniquement les données du recensement de 2013, car la question n'a pas été posée en 2002. Là encore, le modèle Sud est utilisé pour convertir les différentes probabilités de survies ( $\mathrm{n}_{25}$ chez les femmes et ${ }_{n} \mathrm{P}_{35}$ chez les hommes) en un indice unique de mortalité entre 15 et 60 ans $\left(45 q_{15}\right)$. Pour atténuer les fluctuations imputables aux faibles effectifs, nous lissons les estimations indirectes à l'aide d'une moyenne mobile sur 2 ans.

Enfin, les taux de mortalité par âge ont été calculés directement en rapportant les décès déclarés dans les 12 mois ayant précédé le recensement à la population énumérée dans les trois sites. Compte tenu des faibles effectifs, nous n'avons pas cherché à appliquer les méthodes de distribution des décès, mais uniquement à évaluer l'ampleur de la sousdéclaration des décès et examiner si elle varie par âge.

Tableau I : Effectifs de population recensés par groupes d'âge en 2002 et 2013 et différence relative par rapport aux effectifs de résidents dans les observatoires

\begin{tabular}{|l|l|l|l|l|l|l|}
\hline 2002 & Bandafassi & Mlomp & Niakhar \\
\hline 0 & Effectifs recensés' & Différence relative ${ }^{2}$ & $\begin{array}{l}\text { Effectifs } \\
\text { recensés' }\end{array}$ & $\begin{array}{l}\text { Différence } \\
\text { relative }^{2}\end{array}$ & $\begin{array}{l}\text { Effectifs } \\
\text { recensés }^{1}\end{array}$ & $\begin{array}{l}\text { Différence } \\
\text { relative }^{2}\end{array}$ \\
\hline $1-4$ & 371 & $-25.4 \%$ & 105 & $-32.7 \%$ & 944 & $-27.7 \%$ \\
\hline $5-14$ & 1505 & $-1.0 \%$ & 510 & $-7.9 \%$ & 4043 & $-4.2 \%$ \\
\hline $15-59$ & 4966 & $+8.1 \%$ & 1518 & $+5.3 \%$ & 9345 & $+5.7 \%$ \\
\hline $60+$ & 608 & $-9.3 \%$ & 2219 & $-13.5 \%$ & 12393 & $-11.4 \%$ \\
\hline 2013 & & $-15.1 \%$ & 733 & $-10.3 \%$ & 2313 & $-4.6 \%$ \\
\hline 0 & 372 & & & & & \\
\hline $1-4$ & 1673 & $-20.2 \%$ & 113 & $-37.6 \%$ & 1242 & $-29.0 \%$ \\
\hline $5-14$ & 3930 & $-7.6 \%$ & 449 & $-23.5 \%$ & 5787 & $-11.7 \%$ \\
\hline $15-59$ & 5616 & $+4.3 \%$ & 1377 & $-10.9 \%$ & 12917 & $+2.0 \%$ \\
\hline $60+$ & 813 & $-12.6 \%$ & 2414 & $-20.4 \%$ & 16025 & $-23.2 \%$ \\
\hline
\end{tabular}

I Selon les données des recensements (ces totaux n'incluent pas les quelques personnes dont l'âge est inconnu au recensement).

2 Calculée comme $\left(\mathrm{n}_{\mathrm{rgph}}-\mathrm{n}_{\mathrm{obs}}\right) / \mathrm{n}_{\text {obs }}$ où $\mathrm{n}_{\text {rgph }}$ correspond aux effectifs du recensement et nobs aux effectifs de l'observatoire (en excluant, quand cela est possible, les migrants saisonniers)

\section{Résultats}

\section{Structures par âge et sexe des populations résidentes}

Avant d'en venir aux niveaux de mortalité, nous examinons les effectifs de population par âge et sexe, afin de vérifier que la comparaison porte bien sur des populations identiques. Les effectifs de population s'avèrent systématiquement plus élevés selon les données des observatoires (Graphique 2 et Tableau I). Ces différences d'effectifs varient par sexe; à Bandafassi et Niakhar, elles sont plus larges chez les hommes, tandis qu'à Mlomp elles sont plus larges chez les femmes. Ces écarts sont par ailleurs plus importants en 2013 qu'en 2002, pour chaque sexe et dans tous les sites. La comparaison des effectifs entre les deux sources permet également de mettre en évidence un sous-enregistrement très prononcé des enfants de moins d'un an dans le recensement, de $20 \%$ à Bandafassi en 2013 jusqu'à $-38 \%$ à Mlomp. Ce sous-enregistrement caractérise également les enfants de I-4 ans, quoique dans une moindre mesure, tandis que les recensements enregistrent davantage d'enfants de 5 à 14 ans que les observatoires (sauf à Mlomp en 2013). II est possible que des erreurs d'âges soient ici en cause, transférant des enfants de moins de 5 ans dans la catégorie des 5 à 10 ans. Les différences relatives entre effectifs recensés et repris dans les observatoires sont également assez larges chez les adultes de 15 à 60 à Mlomp et à Niakhar, et chez les plus de 60 ans à Bandafassi.

En examinant ces écarts entre observatoires et recensements, il est difficile de dissocier le sousdénombrement de certaines populations et l'effet des différences dans la définition utilisée dans chaque source de données pour caractériser la population résidente. Les critères utilisés pour identifier des résidents sont en effet plus "restrictifs » dans les recensements, car ces derniers couvrent l'ensemble du territoire et doivent veiller à ne pas compter deux fois une même personne à deux endroits différents. Les recensements considèrent donc comme résidents les individus ayant vécu "habituellement" dans le ménage durant les 6 mois précédant le recensement (ainsi que les individus installés depuis moins de 6 mois dans le ménage mais ayant l'intention d'y rester). Les observatoires de population adoptent une définition bien plus large, 
car ils visent à saisir la dynamique démographique des zones suivies, et leur population intègre donc des individus mobiles régulièrement de passage dont la plupart ont le statut de non-résident d'après le recensement. Par exemple, à Niakhar, certaines personnes absentes depuis plus de six mois sont considérées comme résidentes; c'est le cas des travailleurs saisonniers qui ont une épouse ou des enfants dans la zone et qui reviennent au moins une fois tous les six mois. Les élèves ou talibés dont les parents résident dans la zone sont également résidents dans la concession de leurs parents. A Bandafassi, les critères sont encore plus englobants puisque les personnes résidant habituellement dans la concession et les personnes absentes depuis moins de quatre ans sont considérés comme résidents, sauf si l'absence est consécutive à la formation ou à la rupture d'une union.

Nous avons exploité les données recueillies à Bandafassi, Mlomp et Niakhar sur les migrations pour approcher au plus près la définition de la résidence qui prévaut dans les recensements. Pour produire les décomptes présentés dans le Tableau $\mathrm{I}$ et le Graphique 2, nous avons également utilisé les informations collectées sur les absences temporaires à Niakhar et Mlomp. A Bandafassi, les absences ne sont pas suffisamment bien enregistrées pour être utilisées. Ces absences sont cependant connues au moment des différents passages; une personne pourrait effectuer une migration temporaire entre deux passages sans que ce mouvement ne soit connu du système d'enregistrement. Une partie des absences ou migrations saisonnières pourraient également être sous-déclarées, soit parce que les questions ne sont pas systématiquement posées, ou en raison d'erreurs de mémoire. A titre indicatif, la population de Mlomp en 2013 passe de 8633 résidents à 6089 lorsque les migrants saisonniers sont exclus, tandis que celle de Niakhar pour la même année passe de 45517 à 44633 personnes. L'exclusion des migrants saisonniers a donc un effet bien plus large à Mlomp, parce que ces derniers sont plus nombreux mais aussi sans doute mieux repérés.

En définitive, cette comparaison au niveau agrégé ne permet pas de mener une analyse précise de la couverture des recensements, même après exclusion des migrants enregistrés. Les écarts observés aux âges adultes sont vraisemblablement attribuables en partie à des différences de définition. On peut par contre supposer que pour les jeunes enfants et les personnes âgées, il s'agit d'avantage de sousdénombrements. Des erreurs d'âges transférant certains individus d'une classe d'âge à l'autre ne sont pas non plus à exclure. Ces questions devront être réévaluées dans des travaux ultérieurs qui s'appuieront sur des appariements entre sources au niveau individuel.

Au niveau agrégé, on peut déjà utiliser l'indice de Myers pour évaluer la qualité des déclarations sur les âges. Cet indice d'attraction peut être interprété comme une estimation du pourcentage de personnes dont l'âge devrait être corrigé pour effacer l'attraction sur certains âges (Swanson et al. 2004). Dans le recensement de 2002, cet indice s'élevait à 7.7 à Mlomp, 16.I à Bandafassi et 19 à Niakhar. En 2013 , on note une nette amélioration de la qualité des âges déclarés, puisque cet indice s'établit à 5.3 à Mlomp, 6.5 à Bandafassi et 8.8 à Niakhar dans les données du recensement. Dans les observatoires, la valeur de cet indice est partout inférieure à 3 . 
Graphique 2: Structures par âge de la population des trois zones d'études selon la source de données en 2002 et 2013
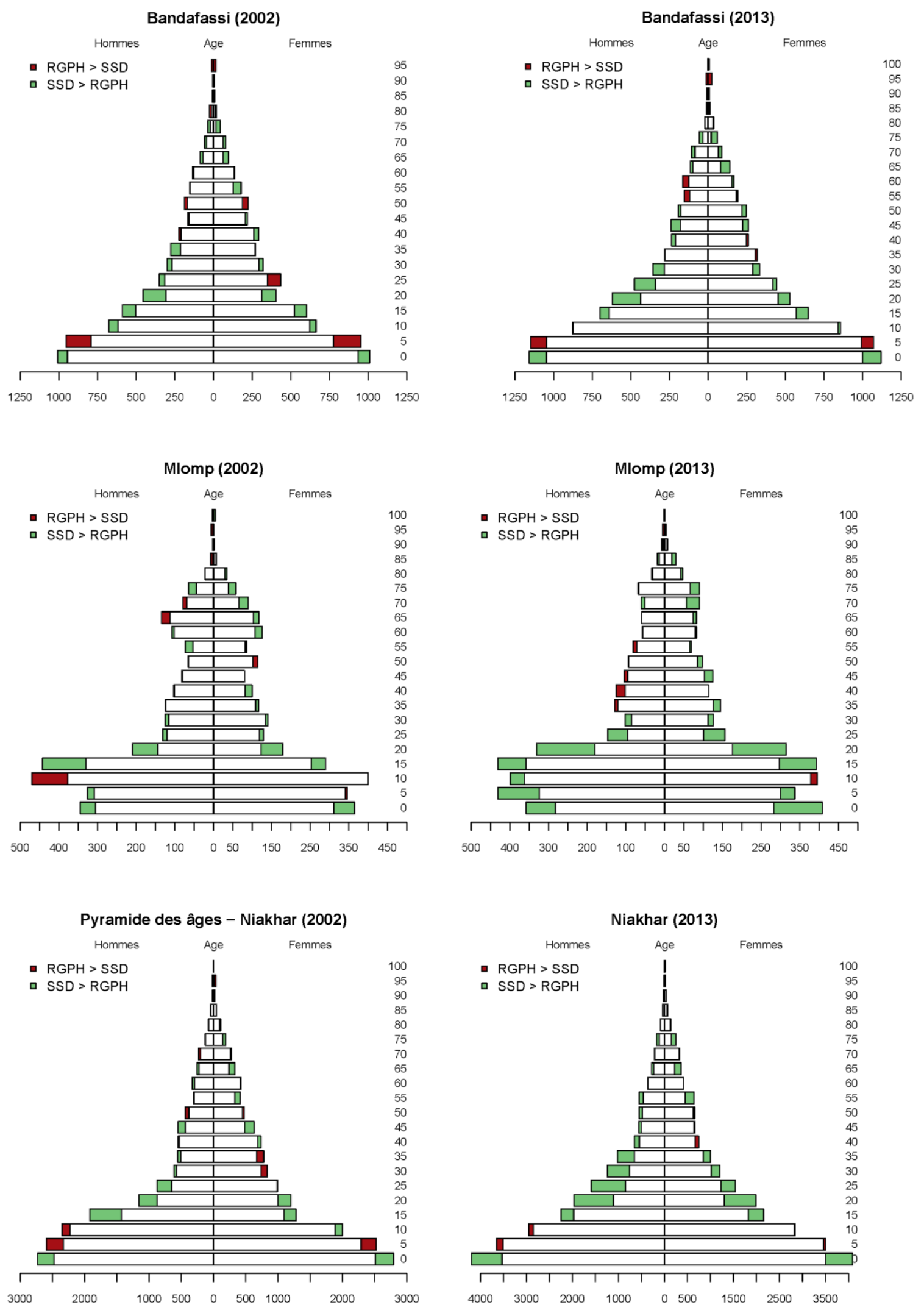

Estimations indirectes de la mortalité des enfants Examinons maintenant les estimations indirectes du niveau de la mortalité infanto-juvénile obtenues à

partir des déclarations des mères (après avoir exclu les migrantes) au regard des niveaux attendus (Graphique 3). 
À Bandafassi, la tendance obtenue de façon indirecte est assez proche de celle réellement observée dans l'observatoire, bien que le niveau de la probabilité ${ }_{5} q_{0}$ soit en moyenne $10 \%$ trop basse dans les deux recensements ${ }^{8}$. À Niakhar, les estimations indirectes sont acceptables jusqu'au milieu des années 2000. L'estimation du recensement est $20 \%$ plus élevée que celle de l'observatoire jusqu'en 1995, et par après, elle est nettement plus basse. Ceci résulte du fait que le pic de mortalité de la fin des années 1990 n'est pas représenté, car les estimations indirectes reposent sur une hypothèse de linéarité des tendances, et en conséquence, elles tendent à lisser les irrégularités. Ce pic de mortalité est imputable à une épidémie de méningite, ainsi qu'à une reprise des fièvres, maladies diarrhéiques et infections respiratoires aiguës (Etard et al. 2004). Les estimations pour les années les plus récentes, basées sur les femmes de moins de 30 ans en 2013, indiquent par contre que la mortalité a connu une hausse, tandis que les données de l'observatoire témoignent d'une baisse rapide et continue de la mortalité des enfants. Là encore, des analyses complémentaires au niveau individuel sont nécessaires pour identifier la source de cet écart; il pourrait s'agir d'erreurs de déclarations dans le recensement mais aussi d'un effet de sélection des répondantes les plus jeunes. Le recensement comptabilise en effet moins de femmes à ces âges que celles enregistrées comme résidentes dans l'observatoire (cela est apparent dans la Figure I). Finalement, les estimations pour Mlomp sont assez erratiques, compte tenu de la plus petite taille de cet observatoire et des niveaux de mortalité et de fécondité plus faibles. Les valeurs présentées ici ont été lissées par moyenne mobile. Mais là aussi, les niveaux obtenus indirectement sont trop faibles, d'environ $40 \%$ dans le cas du recensement de 2002 , et $25 \%$ dans le cas du recensement de 2013, vraisemblablement en raison d'omissions.

Dans les trois sites, les effectifs de femmes considérées pour ce calcul indirect restent faibles; entre 894 femmes à Mlomp en 2002 et 7607 femmes à Niakhar en 2013. A titre de comparaison, les enquêtes EDS menées en Afrique sub-saharienne interrogent en moyenne 10000 femmes pour recueillir des informations sur la survie des enfants ${ }^{9}$. Toutefois, l'impression qui se dégage de l'application de la méthode à ces six extraits de recensement est celle d'une sous-estimation de la mortalité, plutôt que de variations aléatoires liées aux faibles échantillons. En définitive, les estimations indirectes de la mortalité des enfants semblent peu robustes et à considérer avec la plus grande prudence.

Graphique 3 : Comparaison des estimations indirectes de la mortalité des enfants issues des recensements de 2002 et 2013 avec les niveaux obtenus directement des données longitudinales

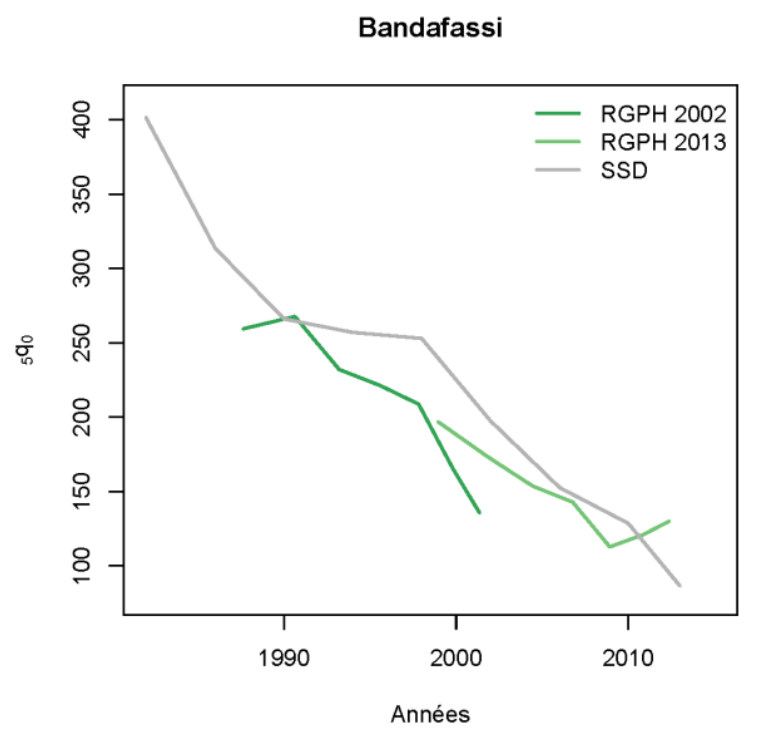

\footnotetext{
8 Par souci de concision, nous ne présentons pas les tableaux associés. L'ensemble des tableaux détaillés et les données anonymes sont disponibles sur demande aux auteurs.
}

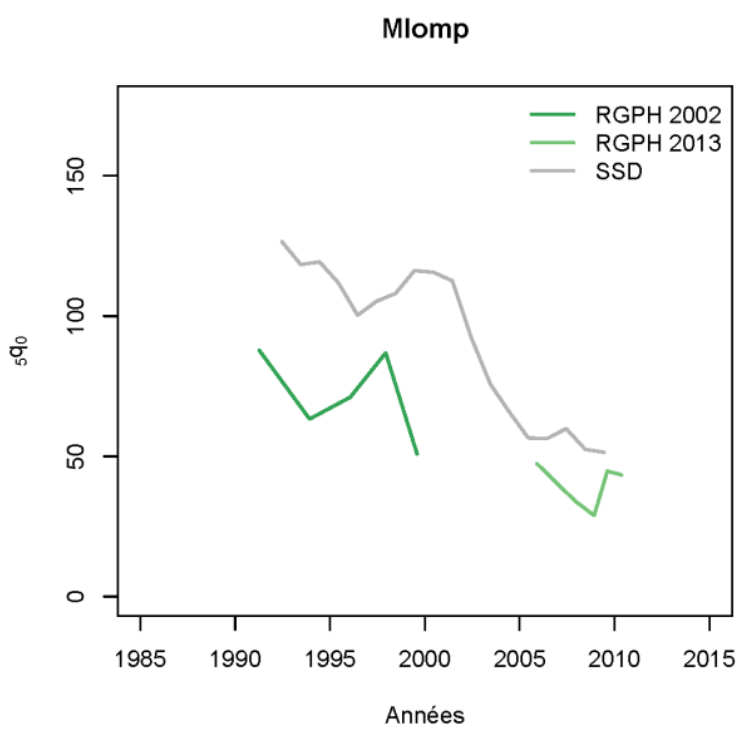

9 Sc:Demographic and health Surveys - STAT COMPILER : http://www.statcompiler.com/ 


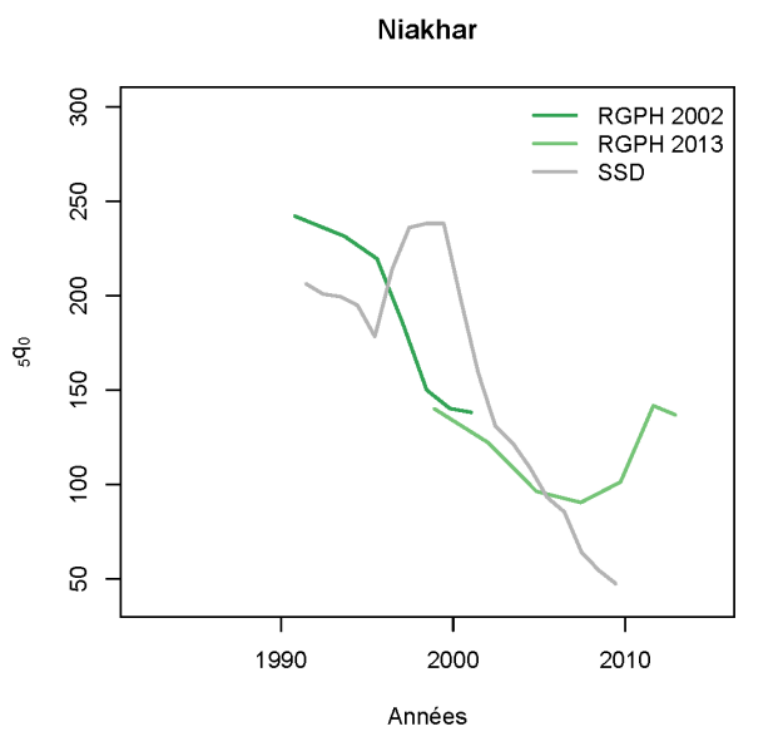

Estimations indirectes de la mortalité des adultes Les niveaux de la probabilité ${ }_{45} q_{15}$ estimés à partir des proportions d'orphelins déclarées dans le recensement de 2013 se révèlent également inférieurs aux niveaux attendus sur la base des données longitudinales (Graphique 4). A Bandafassi, le ratio des estimations indirectes sur les estimations directes s'élève à $84 \%$ en moyenne sur l'ensemble des points considérés chez les femmes, mais il chute à $48 \%$ chez les hommes, en partie en raison des valeurs particulièrement basses calculées à partir des jeunes de 10-19 ans. A Mlomp, les tendances et les différentiels par sexe obtenus indirectement sont plus proches des valeurs attendues, mais la mortalité reste sous-estimée de l'ordre de $35 \%$ pour les deux sexes. A Niakhar les valeurs calculées à partir de la survie des mères sont inférieures de $25 \%$ aux valeurs attendues, et la sous-estimation se chiffre à $50 \%$ dans le cas des pères. En définitive, dans aucun des sites cette méthode ne permet de générer des estimations de mortalité utilisables.

Comme précisé précédemment, la source de biais généralement la plus citée dans le cadre de la méthode des orphelins est l'« effet d'adoption", soit la confusion entre parents biologiques et parents adoptifs. II existe toutefois très peu d'études ayant cherché à évaluer directement cet effet d'adoption et leurs résultats ne sont pas univoques. Une enquête réalisée à Bandafassi, peu après le recensement local des Peuls Bandé en 1975, a montré que près d'un tiers des enfants de 10 à 14 ans qui étaient orphelins de pères avaient en fait été déclarés comme ayant toujours un père survivant lors de ce recensement (Pison and Langaney 1985). Aucune erreur de déclaration n'avait été observée pour les mères. Par contre, au Zimbabwe, Robertson et al. (2008) ont analysé, à partir des données longitudinales de l'observatoire de Manicaland, la cohérence des déclarations faites sur la survie des parents entre trois passages, et constaté que la survie des pères était mieux déclarée. Environ un tiers des enfants de moins de 16 ans déclarés comme orphelins de mère au premier passage furent ensuite déclarés comme non-orphelins lors d'au moins un des deux passages qui ont suivi, et ce fut le cas d'un enfant sur sept parmi ceux déclarés comme orphelins de père. Les autres travaux disponibles sur ce thème identifient le biais d'adoption en comparant des estimations modélisées du nombre d'orphelins avec des observations issues de recensements ou d'enquêtes (Grassly et al. 2004), où en comparant différentes séries d'estimation entre elles (Timaeus 1986). Ils concluent que le biais d'adoption est surtout apparent à partir des niveaux de mortalité les plus récents, obtenus des déclarations relatives aux plus jeunes enfants, et que le biais est également plus prononcé pour les mères. Or aucun de ces deux phénomènes n'est observé ici dans les sites sénégalais; les estimations masculines sont particulièrement basses et les écarts avec les valeurs attendues sont tout aussi élevés pour les périodes les plus anciennes. On semble donc plutôt être en présence d'une incompréhension générale de la question, à laquelle participe certainement le fait que les termes associés à la filiation sont rarement réservés aux parents biologiques. Diop (1985) souligne qu'en wolof, le terme "baay" (père en wolof) s'étend au père, aux frères, aux cousins, et par extension, à tous les hommes de la même génération (à l'exception de l'oncle maternel). 
Graphique 4 : Comparaison des estimations indirectes de la mortalité des adultes issues des recensements de 2013 avec les niveaux obtenus directement des données longitudinales
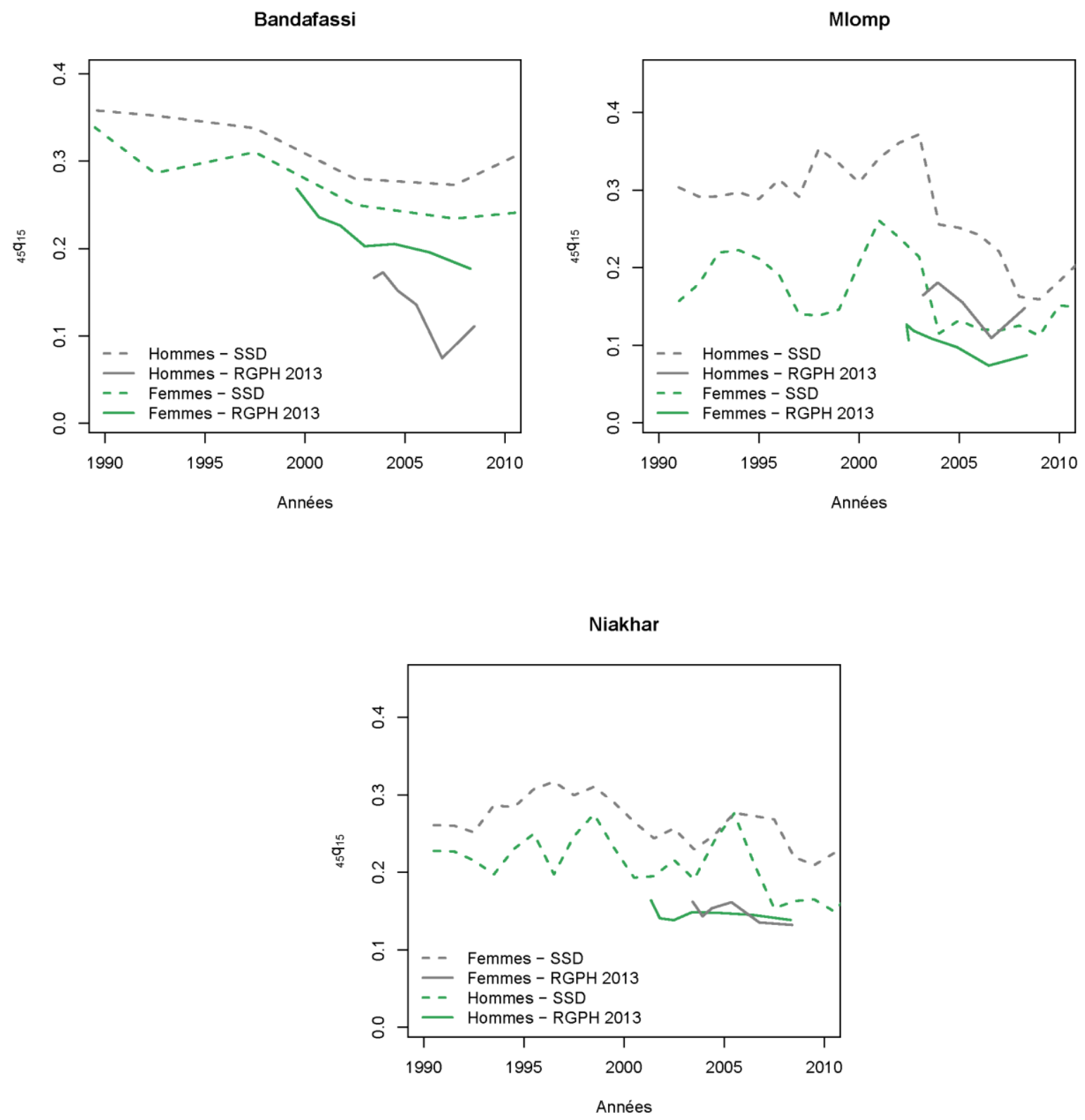

Des erreurs d'âge pourraient également avoir contribué à biaiser les estimations. En particulier, l'attirance sur les âges ronds $(5,10,15)$ va induire une sous-estimation de la mortalité ${ }^{\prime 0}$. Des transferts systématiques, dans le sens de «vieillissements » ou "rajeunissements», sont possibles également. La présence d'erreurs sur les âges peut s'observer en

10 Par exemple, les proportions de non-orphelins ne sont pas modifiées si une certaine proportion d'enfants de 6 et 7 ans est enregistrée comme ayant 5 ans. Par contre, si un certain nombre d'enfants âgés de 3 à 4 ans sont déplacés vers 5 ans, ils vont transférer vers un autre groupe d'âge des risques de mortalité plus faibles. comparant les proportions de parents survivants déclarée par sexe: ces dernières s'avèrent souvent plus élevées lorsqu'elles sont rapportées par les hommes que lorsqu'elles sont rapportées par les femmes. Nous l'observons nettement dans deux des trois sites: chez les adultes de 40-44 ans, les proportions de mères survivantes sont supérieures de $8 \%$ chez les hommes à Mlomp, et de $7 \%$ à Niakhar. A Bandafassi, elles sont quasiment identiques. Ceci semble indiquer que les femmes ont été davantage "rajeunies" que les hommes, ou que les hommes ont été davantage "vieillis". Un vieillissement artificiel va provoquer une sous- 
estimation importante de la mortalité, puisqu'il conduit à surestimer la durée d'exposition des parents au risque de mourir.

Tables de mortalité déduites des informations sur les décès des douze derniers mois

L'introduction de questions sur les décès survenus dans les ménages au cours des 12 derniers mois dans les deux recensements permet de calculer des tables de mortalité. Les niveaux de mortalité infantile $\left(ı q_{0}\right)$, juvénile $\left(4 q_{1}\right)$, aux âges adultes $\left(45 q_{15}\right)$ et aux âges élevés (20960) sont présentés (pour les deux sexes confondus) dans le Tableau 2 pour les trois sites, ainsi que les espérances de vie à la naissance $\left(\mathrm{e}_{0}\right)$ et à 5 ans $\left(e_{5}\right)$. Les estimations issues des recensements sont comparées aux niveaux de mortalité obtenus à l'aide des données longitudinales des suivis démographiques (après avoir exclu les migrants saisonniers à Niakhar et Mlomp). Les tables de mortalité ne peuvent être calculées pour Mlomp sur une seule année (en 20/3), le nombre de décès annuel étant trop faible - la population est de plus petite taille et les niveaux de mortalité sont plus bas que dans les autres sites. En 2002 par contre, un calcul est possible, car la mortalité de cette année a été particulièrement élevée. Des dizaines de résidents de l'observatoire sont décédés le 26 Septembre 2002, soit deux mois avant le recensement, dans le naufrage du Joola, un ferry reliant Ziguinchor à Dakar, qui a chaviré au large de la côte de la Gambie, coûtant la vie à 1863 personnes au total.

Ces tables de mortalité restent entachées d'erreurs aléatoires: en 2002, le recensement a comptabilisé seulement 100 décès à Mlomp, et 293 à Niakhar, par exemple. II faut également garder à l'esprit que les populations considérées varient; elles sont plus larges selon les observatoires et il est concevable que certains décès enregistrés dans ces observatoires incluent des individus qui ne devraient pas être considérés comme résidents selon la définition des recensements.

Tableau 2: Indices de mortalité par âge obtenus à partir des déclarations sur les décès des 12 derniers mois dans les recensements et selon les données longitudinales des observatoires de population

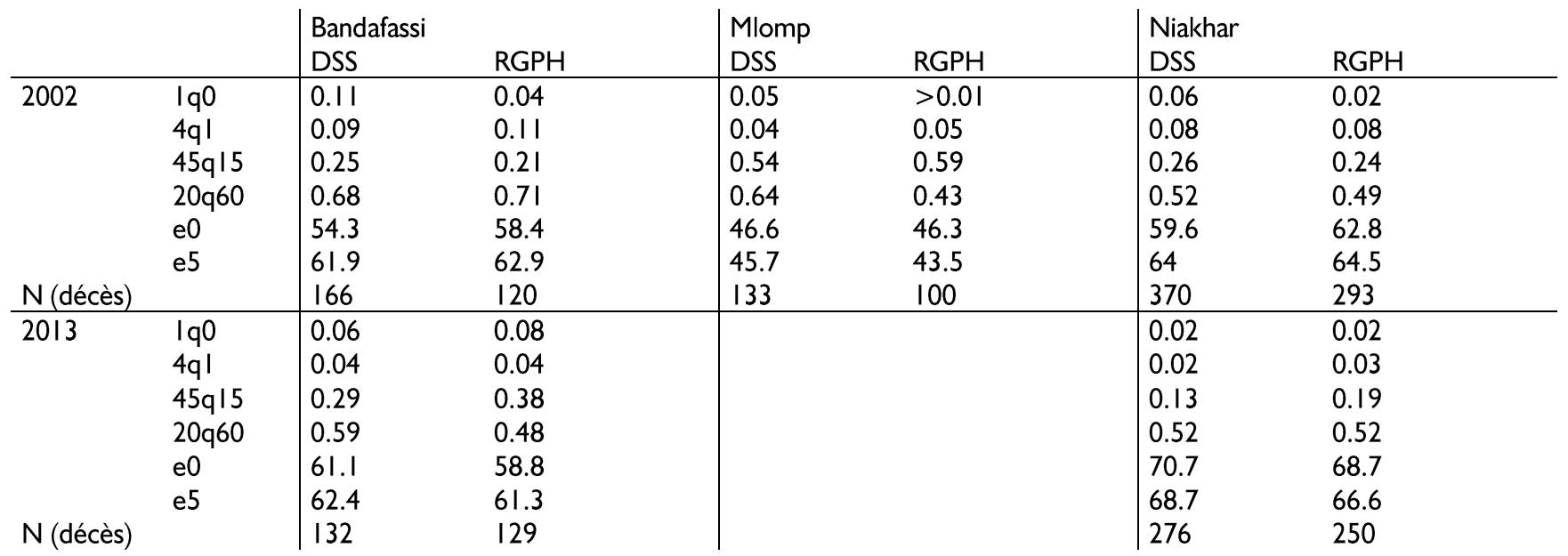

L'analyse des tables de mortalité obtenues à l'aide des deux sources de données met toutefois en évidence des schémas similaires dans les trois sites. Les informations recueillies dans le recensement ont donné lieu à une sous-estimation sensible de la mortalité infantile dans le recensement de 2002. C'est moins le cas en 2013 à Bandafassi et à Niakhar. Par contre, la mortalité juvénile est partout assez bien estimée, sans doute en raison d'erreurs sur les âges aux décès qui prennent la forme d'une attirance sur I an, et conduisent à sous-estimer la mortalité infantile tout en rehaussant la mortalité juvénile. Un constat comparable avait déjà été fait par Pison et al. (1995) à partir du recensement sénégalais de 1988. Les décès de moins d'un an semblaient également sensiblement sous-déclarés en 1988, tandis que la mortalité entre $I$ et 5 ans avait été considérée comme fiable.

Entre 15 et 60 ans les données du recensement tendent à procurer des niveaux de mortalité très proches de ceux enregistrés dans le suivi, voire même légèrement plus hauts en 2013. II est possible que certains décès déclarés fassent référence à des migrants, ou que des décès aient été déclarés plusieurs fois dans différents ménages. Entre 60 et 80 ans, compte tenu des omissions plus fréquentes des personnes âgées (et de leurs décès) ainsi que des erreurs sur les âges, la concordance entre les estimations du recensement et celles du suivi est remarquable. Dans l'ensemble, ces résultats confirment que les données portant sur les décès survenus dans les ménages ne sont pas adaptées à 
l'analyse de la mortalité des enfants. De façon plus inattendue, ils indiquent que les taux obtenus de façon directe au-delà de 5 ans procurent des niveaux de mortalité tout à fait plausibles, sans nécessairement procéder à des ajustements. Les espérances de vie à 5 ans sont très proches, avec des écarts de moins de 2 ans dans tous les cas (sauf à Mlomp en 2002). Le recours aux méthodes de distribution des décès (p.ex. la generalized growth balance method) pourrait s'avérer problématique ici, car l'enregistrement des décès n'est pas constant par âge, même aux âges adultes. Le recours à ces méthodes est également contre-indiqué en raison de l'intensité des mouvements migratoires à cette échelle locale, particulièrement à Mlomp.

\section{Discussion}

Dans les pays à faible revenu tel que le Sénégal, les enquêtes rétrospectives à large échantillons et les recensements restent les principales sources de données permettant d'estimer les niveaux et tendances de la mortalité. En dépit d'un investissement matériel et humain considérable et d'une grande préparation technique, les recensements présentent en particulier des risques d'être affectés par des erreurs de déclarations, en raison de l'ampleur de la collecte. Notre étude utilise trois observatoires situés dans des zones rurales au Sénégal comme référence pour identifier certains schémas d'erreurs dans les recensements et en évaluer l'impact sur les estimations de la mortalité.

Cette analyse menée au niveau agrégé permet de relativiser quelques idées assez fréquentes relatives à la qualité des données des recensements africains. Elle montre qu'au-delà des premiers âges de l'enfance, les décès déclarés dans les 12 mois précédant le recensement peuvent procurer des niveaux de mortalité peu biaisés. Par contre, les estimations indirectes de mortalité des moins de cinq ans sous-estiment la mortalité ou, dans le cas de Niakhar en 2013, procurent des tendances peu vraisemblables. Les niveaux obtenus indirectement à partir de la survie des parents sont également nettement trop bas, en particulier pour les pères. Cette observation cadre avec les résultats obtenus par Pison et Langaney (1988) sur Bandafassi dans les années 1970s, mais pas avec ceux de Robertson et al. (2008), qui ont montré que le biais d'adoption est plus prononcé chez les mères. En définitive, sur base des analyses existantes, il n'est pas possible d'établir si les données sont généralement de meilleure qualité quand elles concernent la survie des mères. Des analyses complémentaires sont nécessaires sur ce thème, car les informations sur la survie des parents permettent non seulement de mesurer la mortalité, mais également de documenter les conditions de vie des orphelins.

La principale limite de notre étude est que nous comparons deux sources de données sur une base géographique, plutôt qu'au niveau individuel. Par conséquent, les ménages entiers pourraient être inclus dans une source sans être inclus dans la seconde. Des appariements individuels sont en cours, sur la base des noms et prénoms des résidents des observatoires, et des relations de parenté qui associent les membres d'un même ménage. Ces appariements contribueront à affiner ces premières analyses, notamment en excluant tout à fait les migrants saisonniers et en évaluant la qualité des déclarations au niveau individuel. Ils permettront par exemple d'explorer plus en détail les caractéristiques des résidents qui ont été omis au recensement, ou d'analyser la qualité des données sur les parités, la survie des parents, et les décès maternels. Une seconde limite tient au fait que des problèmes de qualité des données restent possibles également dans les observatoires, tels que des omissions d'événements, de ménages, des problèmes de double compte des migrants, etc. Par ailleurs, la définition de la population suivie est parfois problématique, surtout dans les cas où la collecte des migrations saisonnières semble incomplète, comme à Niakhar et à Bandafassi. Enfin, il faut préciser que les effectifs considérés restent relativement faibles (bien qu'au total, les trois observatoires concernent une population de plus de 60000 personnes en 2013). Afin de confirmer ou non les conclusions tirées de cette première évaluation, des analyses comparables pourraient être menées dans d'autres observatoires qui fonctionnent dans des pays où des recensements ont été récemment organisés.

\section{Remerciements}

Cette analyse s'inscrit dans le cadre du projet MADAS, financé par l'Agence Nationale de la Recherche (ANR) en France (ANR-II-BSHI-0007 coordonné par G. Pison). Nous remercions Laetitia Douillot, Clémentine Moerman et Lucie Lecomte pour le travail réalisé sur les bases de données des trois observatoires. Nos remerciements s'adressent également à Pape Niokhor Diouf et Paul Senghor.

\section{Bibliographie}

Adjuik, M., Smith, T., Clark, S., Todd, J., Garrib, A., Kinfu, Y., Kahn K., Mola, M., Ashraf, A., Masanja, $\mathrm{H}$. et al. (2006) Cause-specific mortality rates in sub-Saharan Africa and Bangladesh. Bulletin of the World Health Organization, 84 (3): |8|-|88.

Alkema, L., New, J.R., Pedersen, J., You, D., Bastian, P., Wu, J., Wardlaw, T., Mathers, C., Boerma, T., Ho, J., Suzuki, E., Pelletier, F., Andreev, K., 
Gerland, P., Gu, D., Li, N., Sawyer, C., Spoorenberg, T., Wilmoth, J., Hill, K., Alkema, L., Cousens, S., Croft, T., Jones, G., Guillot, M., Pedersen, J., \& Walker, N. (2014) Child mortality estimation 2013: an overview of updates in estimation methods by the United Nations Interagency Group for Child Mortality Estimation. PLOS ONE, 9 (7): el0III 2.

Bennett, N. \& Horiuchi, S. (1984) Mortality estimation from registered deaths in less developed countries. Demography, 2I(2): 2I7234.

Blacker, J. (1984). Experience in the use of special mortality questions in multi-purpose surveys: the single-round approach. In United Nations (ed.), Data bases for mortality measurement, chapter IX, pages 79-89. United Nations, New York.

Brass, W. \& Bamgboye, E. (I98I) The time location of reports of survivorship: estimates for maternal and paternal orphanhood and the ever-widowed. Centre for Population Studies Research Paper, 8II.

Delaunay, V., Douillot, L., Diallo, A., Dione, D., Trape, J-F., Medianikov, O., Raoult, D., \& Sokhna, C. (20/3). Profile: The Niakhar Health and Demographic Surveillance System. International Journal of Epidemiology, 42 (4): I002-10। I.

Desgrées du Loû, A., Pison, G., Samb, B. \& Trape, JF. (1996) L'évolution des causes de décès d'enfants en Afrique : une étude de cas au Sénégal avec la méthode d'autopsie verbale. Population, $5 \mathrm{I}$ (4), pages 845-882.

Diop, A. (1985). La famille wolof. Karthala.

Dorrington, R. (2013). The generalized growth balance method. In Moultrie, T., Dorrington, R., Hill, A., Hill, K., Timæus, I. \& Zaba B. (ed), Tools for demographic estimation. Paris: International Union for the Scientific Study of Population/UNFPA.

Duthé, G. (2008). Malaria Resurgence in Senegal: Measuring Malaria Mortality in Mlomp. Population, 63 (3): 443-468.

Etard, J-F., Le Hesran, J. Y., Diallo, A., Diallo, J.P. , Ndiaye, J. L. \& Delaunay, V. (2004). Childhood mortality and probable causes of death using verbal autopsy in Niakhar, Senegal, 1989-2000. Int J Epidemiol, 33 (6): | 286-1292

Ewbank, D. (198I). Age misreporting and ageselective underenumeration: Sources, patterns and consequences for demographic analysis. Committee on Population and Demography, Report No. 4.

Grassly, N., Lewis, J., Mahy, M., Walker, N., \& Timaeus, I (2004). Comparison of householdsurvey estimates with projections of mortality and orphan numbers in sub-Saharan Africa in the era of HIV/AIDS. Population Studies, 58:2: 207-2I 7.

Hill, K. (1977). Estimating Adult Mortality Levels from Information on Widowhood. Population Studies, 3I (I): 75-84.

Hill, K. (1987). Estimating Census and Death Registration Completeness. Asian and Pacific Population Forum, I(3): 8-I3.

Hill, K. (20I3). Indirect estimation of child mortality. In Moultrie, T., Dorrington, R., Hill, A., Hill, K., Timæus, I. \& Zaba B. (ed), Tools for demographic estimation. Paris: International Union for the Scientific Study of Population/UNFPA.

Hill, K. \& Stanton, C. (20II). Measuring maternal mortality through the census: rapier or bludgeon? Journal of Population Research, 28 (I): 3I-47.

Masquelier, B. (2010). Estimation de la mortalité adulte en Afrique subsaharienne à partir de la survie des proches. Apports de la microsimulation. $\mathrm{PhD}$ dissertation. Presses Universitaires de Louvain.

Masquelier, B. (20I4). Taille des fratries et taille des familles dans les données d'enquêtes utilisées pour estimer la mortalité. Population, 69 (2): 249-268.

Masquelier, B., Reniers, G. \& Pison, G. (20|4). Divergences in mortality trends in sub-Saharan Africa: survey evidence on the survival of children and siblings. Population Studies, 68 (2): I6I-I77.

Moultrie, T., Dorrington, R., Hill, A., Hill, K., Timæus, I. \& Zaba B. (20|3), Tools for demographic estimation. Paris: International Union for the Scientific Study of Population/UNFPA.

Pison, G. (2005). Population observatories as sources of information on mortality in developing countries. Demographic Research, I3 (I3): 30I334.

Pison, G. and Langaney, A. (1985). The Level and Age Pattern of Mortality in Bandafassi (Eastern Senegal): Results from a Small-Scale and Intensive Multi-Round Survey. Population Studies, 39 (3): pp. 387-405.

Pison, G. and Langaney, A. (1988). Age Patterns of Mortality in Eastern Senegal : Comparison of Micro and Survey Approaches. In Caldwell, J., Hill, A. \& Hull (ed), Micro-approaches to demographic research, pages 297-317. Kegan Paul International, London.

Pison, G., Hill, K., Cohen, B. \& Foote, K. (1995). Population Dynamics of Senegal. National Academy Press, Washington.

Pison, G., Gabadinho, A., Wade, A. \& Enel, C. (2002). Mlomp demographic surveillance system, Senegal. In Population and health in developing countries, pages 27I-278. International Development Research Centre. 
Pison, G., Douillot, L., Kante, M., Ndiaye, O., Diouf, P., Senghor, P., Sokhna, C. \& Delaunay, V. (2014). Health \& Demographic Surveillance System Profile: Bandafassi Health and Demographic Surveillance System (Bandafassi HDSS), Senegal. International Journal of Epidemiology, 43 (3): 739-748.

Preston, S. \& Bennett, N. (1983). A census-based method for estimating adult mortality. Population Studies, 37(I): 9I-104.

Robertson, L., Gregson, S., Madanhire, C., Walker, N., Mushati, P., Garnett, G., \& Nyamukapa, C. (2008). Discrepancies between UN models and DHS survey estimates of maternal orphan prevalence: insights from analyses of survey data from Zimbabwe. Sexually Transmitted Infections, $84 \mathrm{~S}(\mathrm{I}): 57-62$.

Swanson, D., Siegel, S.J \& Shyrock H. (2004). The Methods and Materials of Demography. Emerald Group Publishing, San Diego, CA and London.
Timaeus, I. (1986). An Assessment of Methods for Estimating Adult Mortality from Two Sets of Data on Maternal Orphanhood. Demography, 23, 3: 435-450.

Timaeus, I. (|99|). Measurement of adult mortality in less developed countries: A comparative review. Population Index, 57 (4): pp. 552-568.

Timæus, I. (1992). Estimation of adult mortality from paternal orphanhood: a reassessment and a new approach. Population Bulletin of the United Nations, 33: 47-63.

United Nations (20I5). World Population Prospects: The 2015 Revision, Key Findings and Advance Tables. 2015.

Ye, Y., Wamukoya, M., Ezeh, A., Emina, J., \& Sankoh, O. (20/2). Health and demographic surveillance systems: a step towards full civil registration and vital statistics system in subSahara Africa? BMC Public Health, I2 (I): 74I. 